\title{
BMJ Open Does nephrotic syndrome without chronic kidney disease increase the risk of Parkinson's disease and secondary parkinsonism? A nationwide population-based study in Taiwan
}

\author{
Zheng-Hao Huang, ${ }^{1,2}$ Hsiang-Cheng Chen, ${ }^{1}$ Yu-Ching Chou, ${ }^{3}$ Cheng-Li Lin, ${ }^{4,5}$ \\ Chia-Hung Kao, ${ }^{6,7}$ Hsin-Yi Lo, ${ }^{8}$ Tse-Yen Yang, ${ }^{9}$ Feng-Cheng Liu ${ }^{1}$
}

To cite: Huang Z-H, Chen H-C, Chou Y-C, et al. Does nephrotic syndrome without chronic kidney disease increase the risk of Parkinson's disease and secondary parkinsonism? A nationwide population-based study in Taiwan. BMJ Open 2018;8:e020821. doi:10.1136/ bmjopen-2017-020821

\section{- Prepublication history for} this paper is available online. To view these files, please visit the journal online (http://dx.doi. org/10.1136/bmjopen-2017020821).

T-YY and F-CL contributed equally.

Received 30 November 2017 Revised 27 April 2018 Accepted 8 June 2018

Check for updates

(C) Author(s) (or their employer(s)) 2018. Re-use permitted under CC BY-NC. No commercial re-use. See rights and permissions. Published by BMJ.

For numbered affiliations see end of article.

Correspondence to Dr. Tse-Yen Yang; yang_t_y@yahoo.com.tw and Dr. Feng-Cheng Liu; Ifc10399@yahoo.com.tw

\section{ABSTRACT}

Objectives Previous research has shown that patients with nephrotic syndrome (NS) have a higher risk of cognitive impairment, dementia or neurodegenerative disorder. The present study aimed to examine a relationship, if any exists between NS and Parkinson's disease (PD), a neurodegenerative disorder and secondary parkinsonism (sPS).

Methods A nationwide retrospective observational study conducted using data from the 2000-2010 Taiwan National Health Insurance Research Database. This study included 3663 patients with NS and 14652 randomly selected, age-matched and sex-matched patients without NS. A Cox multivariable proportional hazards model was used to evaluate the risk of PD and SPS (PDsPS) in the NS cohort.

Results This study identified a positive association between NS and the risk of PDsPS in both men and women and in all age groups (adjusted HR 1.51; 95\% Cl 1.37 to 1.66). Compared with patients without NS and comorbidities, those with NS with two or more comorbidities exhibited an 8.23-fold higher risk of PDsPS (95\% Cl 6.22 to 10.9$)$ and patients with NS and one comorbidity exhibited a 2.93-fold higher risk of PDsPS (95\% Cl 2.37 to 3.63 ).

Conclusions Patients with NS have an increased risk of PDsPS. This increased risk may be related to brain vascular damage or blood-brain barrier impairment. Further research is necessary to explore the underlying relationship between NS and PDsPS.

\section{INTRODUCTION}

Parkinsonism (PS) is a common movement disorder and prominent bradykinesia with extrapyramidal symptoms. It is most commonly caused by Parkinson's disease (PD), ${ }^{1}$ also known as idiopathic PD. Secondary PS (sPS) is a form of PS caused by trauma, vascular damage, genetic factors, pharmaceutical drugs, infections or other underlying diseases. ${ }^{2-4}$ Although PD is idiopathic, ageing, genetic factors and numerous

\section{Strengths and limitations of this study}

- We used a large sample size that spanned over 10 years.

- Data were from Taiwan's National Health Insurance Research Database (NHIRD) with about $99 \%$ of the Taiwanese population (around 23 million people) enrolled.

- The NHIRD lacks information on patient behaviours and other risk factors, and the disease diagnoses might not accurately reflect patients' medical conditions.

environmental factors might play a role in the pathogenesis of $\mathrm{PD}^{5}$ The prevalence of PD is approximately $0.3 \%$ in the general population and approximately $1 \%$ in people older than 60 years. ${ }^{6}$ The average incidence of PD in Taiwan is approximately 28.8-35.3 per 100000 persons, and the incidence rate is higher in men and the elderly. ${ }^{7}$ However, despite the information available on PD, there are limited data on the prevalence of PS in Taiwan. Epidemiological studies have shown that chronic kidney disease $(\mathrm{CKD}){ }^{8}$ head injury, ${ }^{9}$ depression, ${ }^{10}$ dementia, ${ }^{11}$ stroke, ${ }^{12}$ chronic obstructive pulmonary disease (COPD) ${ }^{13}$ diabetes ${ }^{14}$ and hyperten$\operatorname{sion}^{15}$ are associated with PD and likely also with PS. Increased inflammation has been associated with the degeneration of dopaminergic neurons in patients with PD. ${ }^{16}$

Nephrotic syndrome (NS) is characterised by heavy proteinuria (protein excretion $>3.5 \mathrm{~g} / 24$ hours), hypoalbuminaemia and peripheral oedema. Altered urinary protein levels are a marker of kidney damage and impaired renal function, ${ }^{17}$ which has been associated with cognitive problems. ${ }^{18}{ }^{19}$ Similarly, proteinuria is considered a predictor 
of organ damage and a risk factor for brain, renal and cardiovascular diseases. ${ }^{20}$ Furthermore, community-based studies have demonstrated that proteinuria can increase the risk of dementia. ${ }^{21}{ }^{22}$ Most notably, proteinuria with CKD may be associated with the incidence of PD. ${ }^{8}$

The mechanism by which NS leads to central nervous system (CNS) involvement in the development of PD and sPS (PDsPS) remains unclear. However, the impairment of the blood-brain barrier and proteinuria-related vascular brain damage might play a key role. ${ }^{23}{ }^{24}$ We hypothesise that NS, which may present with normal renal function or early-stage renal disease, also plays a role in PDsPS development. To our knowledge, no study has yet identified a correlation between NS and PDsPS. Therefore, we conducted this study to determine the risk of PDsPS in patients with NS and to identify related risk factors.

\section{METHODS}

\section{Data source}

Data analysed in this retrospective cohort study were retrieved from the Longitudinal Health Insurance Database 2000 (LHID2000). Taiwan established a compulsory insurance programme, the National Health Insurance (NHI) programme, in March 1995, to provide healthcare to nearly $99 \%$ of the 23.74 million residents. ${ }^{25}$ The details of the LHID2000 and NHI programme have been provided in previous studies. ${ }^{26} 27$ In brief, the LHID2000 contains 1000000 randomly selected individuals from the year 2000 registry for beneficiaries, and the data are renewed annually. Disease criteria were defined according to the International Classification of Diseases, Ninth Revision, Clinical Modification (ICD-9-CM). This study was approved and the manuscript was written according to the Strengthening the Reporting of Observational Studies in Epidemiology recommendations.

\section{Study population}

Patients aged $\geq 20$ years with a new diagnosis of NS (ICD-9-CM code 581) from 2000 to 2010 were selected from the LHID2000. The index date was defined as the date of NS diagnosis. NS patients with a history of PDsPS (ICD-9-CM code 332 PD, including ICD-9-CM 332.0 Paralysis agitans and 332.1 sPS) before the index date and CKD (ICD-9-CM code 585) before the end date were excluded. The non-NS cohort was constructed for the same 2000-2010 period, by applying the same exclusion criteria as that of the NS cohort. The index dates for the patients in the non-NS cohort were randomly assigned. Four non-NS controls were frequency-matched to each NS patient according to age (5-year span), sex and index year (table 1).

\section{Patient and public involvement}

Patients and/or the public were not involved in this study.
Table 1 Characteristics of patients between patients with/ without nephrotic syndrome

\begin{tabular}{|c|c|c|c|c|c|}
\hline & \multicolumn{4}{|c|}{ Nephrotic syndrome } & \multirow[b]{4}{*}{$P$ values } \\
\hline & \multirow{2}{*}{\multicolumn{2}{|c|}{$\begin{array}{l}\text { Yes } \\
(n=3663)\end{array}$}} & \multirow{2}{*}{\multicolumn{2}{|c|}{$\begin{array}{l}\text { No } \\
(n=14652)\end{array}$}} & \\
\hline & & & & & \\
\hline & $\mathrm{n}$ & $\%$ & $n$ & $\%$ & \\
\hline Age, year & & & & & 0.99 \\
\hline $20-49$ & 1890 & 51.6 & 7560 & 51.6 & \\
\hline $50-64$ & 1043 & 28.5 & 4172 & 28.5 & \\
\hline$\geq 65$ & 730 & 19.9 & 2920 & 19.9 & \\
\hline Mean $(\mathrm{SD})^{*}$ & 50.2 & 15.8 & 49.7 & 16.0 & 0.14 \\
\hline Gender & & & & & 0.99 \\
\hline Female & 1525 & 41.6 & 6100 & 41.6 & \\
\hline Male & 2138 & 58.4 & 8552 & 58.4 & \\
\hline \multicolumn{6}{|l|}{ Comorbidity } \\
\hline Diabetes & 472 & 12.9 & 980 & 6.69 & $<0.001$ \\
\hline Hypertension & 1251 & 34.2 & 3625 & 24.7 & $<0.001$ \\
\hline Hyperlipidaemia & 1057 & 28.9 & 2305 & 15.7 & $<0.001$ \\
\hline $\begin{array}{l}\text { Coronary artery } \\
\text { disease }\end{array}$ & 636 & 17.4 & 1742 & 11.9 & $<0.001$ \\
\hline Head injury & 117 & 3.19 & 368 & 2.51 & 0.02 \\
\hline COPD & 449 & 12.3 & 1259 & 8.59 & $<0.001$ \\
\hline Depression & 190 & 5.19 & 531 & 3.62 & $<0.001$ \\
\hline Stroke & 89 & 2.43 & 429 & 2.93 & 0.10 \\
\hline Dementia & 37 & 1.01 & 147 & 1.00 & 0.97 \\
\hline Epilepsy & 31 & 0.85 & 96 & 0.66 & 0.21 \\
\hline \multicolumn{6}{|l|}{ Medication } \\
\hline $\begin{array}{l}\text { Immunosuppressant } \\
\text { therapies }\end{array}$ & 95 & 2.59 & 138 & 0.94 & $<0.001$ \\
\hline $\begin{array}{l}\text { Biological therapy } \\
\text { rituximab with } \\
\text { prednisolone } \dagger\end{array}$ & 2 & 0.05 & 6 & 0.04 & 0.66 \\
\hline
\end{tabular}

$\mathrm{X}^{2}$ test.

*t-test.

†Fisher exact test.

COPD, chronic obstructive pulmonary disease.

\section{Outcome, comorbidities and medications}

Both the NS and non-NS cohorts were followed up from the index date until the PDsPS onset date, withdrawal from the NHI programme or the end of 2011, whichever occurred first. The possible reasons for the discontinuity of NHI include death, withdrawal of insurance, immigration or prison sentence. Baseline comorbidities considered in this study were diabetes (ICD-9-CM code 250), hypertension (ICD-9-CM codes 401-405), hyperlipidaemia (ICD-9-CM code 272), coronary artery disease (ICD-9-CM codes 410-414), head injury (ICD-9-CM codes $310.2,800,801,803,804,850,851,853$ and 854), depression (ICD-9-CM codes 296.2, 296.3, 296.82, 300.4 and 311), stroke (ICD-9-CM codes 430-438), COPD (ICD-9-CM codes 491, 492 and 496), dementia (ICD-9-CM codes 290, 294.1 and 331.0) and epilepsy (ICD-9-CM code 345). The use of immunosuppressant 
Table 2 Nephrotic syndrome increased the incidence and HR of Parkinson's disease and secondary parkinsonism among different gender, age, medication and following duration

\begin{tabular}{|c|c|c|c|c|c|c|c|c|}
\hline \multirow[b]{3}{*}{ Outcome } & \multicolumn{6}{|c|}{ Nephrotic syndrome } & \multirow[b]{3}{*}{ Crude HR (95\% Cl) } & \multirow{3}{*}{$\begin{array}{l}\text { Adjusted HR } \\
(95 \% \mathrm{Cl})\end{array}$} \\
\hline & \multicolumn{3}{|l|}{ Yes } & \multicolumn{3}{|l|}{ No } & & \\
\hline & Event & PY & Rate & Event & PY & Rate & & \\
\hline All & 58 & 24335 & 2.38 & 145 & 97065 & 1.49 & $1.60(1.44 \text { to } 1.77)^{\star \star \star}$ & $1.51(1.37 \text { to } 1.66)^{\star \star \star}$ \\
\hline $\begin{array}{l}\text { Excluded patients of } \\
\text { stroke and dementia }\end{array}$ & 51 & 23875 & 2.14 & 121 & 94635 & 1.28 & $1.67(1.50 \text { to } 1.86)^{\star \star \star}$ & $1.58(1.43 \text { to } 1.74)^{\star \star \star ~}$ \\
\hline \multicolumn{9}{|l|}{ Gender } \\
\hline Female & 23 & 10319 & 2.23 & 58 & 41198 & 1.41 & $1.58(1.34 \text { to } 1.87)^{\star \star \star}$ & $1.50(1.28 \text { to } 1.75)^{\star \star \star}$ \\
\hline Male & 35 & 14016 & 2.50 & 87 & 55867 & 1.56 & $1.60(1.40 \text { to } 1.84)^{\star \star \star}$ & $1.54(1.35 \text { to } 1.75)^{\star \star \star}$ \\
\hline \multicolumn{9}{|l|}{ Age to year } \\
\hline $20-49$ & 10 & 13584 & 0.74 & 7 & 53391 & 0.13 & $5.61(4.80 \text { to } 6.57)^{\star \star \star}$ & $4.75(4.06 \text { to } 5.56)^{\star \star \star}$ \\
\hline $50-64$ & 16 & 6745 & 2.37 & 31 & 27233 & 1.14 & $2.08(1.73 \text { to } 2.51)^{\star \star \star}$ & $2.00(1.66 \text { to } 2.40)^{\star \star *}$ \\
\hline$\geq 65$ & 32 & 4005 & 7.99 & 107 & 16441 & 6.51 & 1.23 (0.98 to 1.53$)$ & 1.15 (0.92 to 1.43$)$ \\
\hline \multicolumn{9}{|l|}{ Medication } \\
\hline \multicolumn{9}{|c|}{ Immunosuppressant therapies } \\
\hline No & 57 & 23674 & 2.41 & 142 & 96005 & 1.48 & $1.63(1.46 \text { to } 1.81)^{\star \star \star}$ & $1.53(1.38 \text { to } 1.68)^{\star \star \star}$ \\
\hline Yes & 1 & 660 & 1.51 & 3 & 1060 & 2.83 & 0.53 (0.21 to 1.38$)$ & - \\
\hline \multicolumn{9}{|c|}{ Biological therapy as rituximab with prednisolone } \\
\hline No & 58 & 24312 & 2.39 & 145 & 97016 & 1.49 & $1.60(1.44 \text { to } 1.77)^{\star \star \star}$ & $1.51(1.37 \text { to } 1.66)^{\star \star \star}$ \\
\hline Yes & 0 & 22 & 0.00 & 0 & 49 & 0.00 & - & - \\
\hline \multicolumn{9}{|l|}{ Follow time to years } \\
\hline$\leq 2$ & 13 & 3734 & 3.48 & 54 & 14989 & 3.60 & 0.97 (0.85 to 1.10$)$ & 0.85 (0.75 to 1.00$)$ \\
\hline$>2$ & 45 & 17263 & 2.61 & 91 & 68723 & 1.32 & $1.97(1.77 \text { to } 2.19)^{\star \star \star}$ & $1.92(1.74 \text { to } 2.13)^{\star \star \star}$ \\
\hline
\end{tabular}

${ }^{*} \mathrm{p}<0.05,{ }^{* *} \mathrm{p}<0.01,{ }^{* * *} \mathrm{p}<0.001$.

Adjusted HR, HR adjusted for age; comorbidities of diabetes, hypertension, hyperlipidaemia, coronary artery disease, head injury, COPD, depression, stroke, dementia and epilepsy; and immunosuppressant therapy and the biological therapy as rituximab with prednisolone; crude $\mathrm{HR}$, relative HR; PY, person-years; rate, incidence rate per 1000 person-years.

therapies and biological therapy rituximab with prednisolone was also analysed in the NS and non-NS cohorts (table 2).

\section{Statistical analysis}

The $\chi^{2}$ test and Student's t-test were used to compare the NS and non-NS cohorts for categorical and continuous variables, respectively. The cumulative incidence curves of PDsPS for the NS and non-NS cohorts were plotted using the Kaplan-Meier method, and the difference was tested using a log-rank test (figure 1). The incidence density rates (per 1000 person-years) for PDsPS were calculated in both cohorts and stratified by sex, age, medication and follow-up period. Univariable and multivariable Cox proportional hazards regression analyses were used to assess the HR and 95\% CI of PDsPS associated with NS, compared with non-NS controls. Moreover, we assessed the joint effect of NS and number of comorbidities on PDsPS. All statistical analyses were performed using SAS V.9.4 software (SAS Institute, Cary, North Carolina, USA) for Windows. All $p$ values were two-tailed, and $p<0.05$ was considered significant.

\section{RESULTS}

The study included an NS cohort of 3663 patients and a non-NS cohort of 14652 patients (table 1). The mean age of participants in the NS and non-NS cohorts were $50.2(\mathrm{SD}=15.8)$ and $49.7(\mathrm{SD}=16.0)$ years, respectively. Both cohorts had similar distributions of age and sex, and most people were aged $\leq 49$ years $(51.6 \%)$ and were men (approximately 58.4\%). Comorbidities of diabetes, hypertension, hyperlipidaemia, coronary artery disease, head injury, COPD, depression, stroke, dementia and epilepsy were more prevalent in the NS cohort than in the non-NS cohort $($ all $\mathrm{p}<0.05)$. Compared with the non-NS patients, those with NS exhibited a higher prevalence of immunosuppressant therapy use $(2.59 \%$ vs $0.94 \%)$. The mean follow-up period was 6.64 years in the NS cohort and 6.62 years in the non-NS cohort. The Kaplan-Meier plot revealed that the NS cohort exhibited a higher cumulative incidence of PDsPS than did the non-NS cohort (log-rank test, $\mathrm{p}<0.001$; figure 1 ).

The incidence density rates were 2.38 and 1.49 per 1000 person-years in the NS and non-NS cohorts, respectively (table 2). The multivariable Cox model estimated 


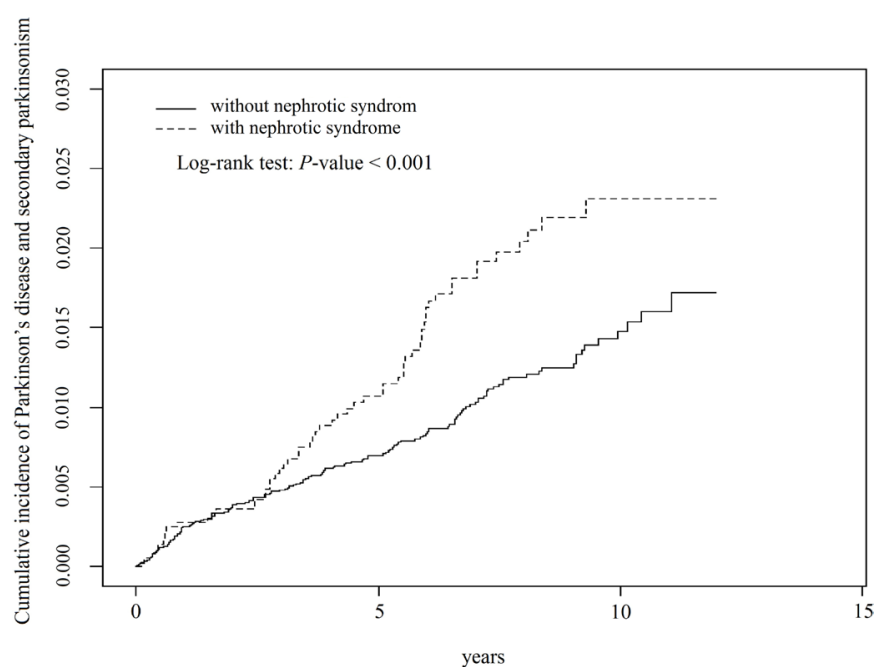

Figure 1 Comparison of cumulative incidence of Parkinson's disease and secondary parkinsonism between patients with and without nephrotic syndrome (NS). The Kaplan-Meier plot revealed that the NS cohort exhibited a higher cumulative incidence of PD than the non-NS cohort did (log-rank test, $p<0.001$ ).

that the adjusted HR (aHR) of PDsPS was 1.51 (95\% CI 1.37 to 1.66 ) in the NS cohort compared with the non-NS cohort. We also performed multivariable Cox analyses while excluding patients with stroke or dementia (3552 patients with NS and 14126 without NS were analysed), in an attempt to more precisely target PD groups. In this additional analysis, the aHR of PDsPS was 1.58 (95\% CI 1.43 to $1.74 ; \mathrm{p}<0.001)$. The sex-specific relative risk of PDsPS was significantly higher in both women $(\mathrm{aHR}=1.50$, $95 \%$ CI 1.28 to 1.75 ) and men (aHR=1.54, 95\% CI 1.35 to 1.75 ) in the NS cohort than in the non-NS cohort. An age-specific analysis revealed that NS patients exhibited a higher risk of PDsPS than did non-NS patients if patients were aged 20-49 years $(\mathrm{aHR}=4.75,95 \%$ CI 4.06 to 5.56) and 50-64 years (aHR=2.00, 95\% CI 1.66 to 2.40). However, this increased risk of PDsPS was not apparent for patients in the $\geq 65$ age group (aHR $=1.15$, 95\% CI 0.92 to 1.43). The aHR of PDsPS was significantly higher in the
NS cohort than in the non-NS cohort for patients without immunosuppressant therapy use (aHR $=1.53$, 95\% CI 1.38 to 1.68). The relative risk of PDsPS was higher in the NS cohort than in the non-NS cohort for patients who did not undergo rituximab and prednisolone biological therapy (aHR $=1.51,95 \%$ CI 1.37 to 1.66 ). The risk of PDsPS in the NS cohort was significantly higher than that in the non-NS cohort when assessed at $>2$ years of follow-up (aHR=1.92, 95\% CI 1.74 to 2.13 ). To address the concern of constant proportionality, we examined the proportional hazard model assumption using a test of scaled Schoenfeld residuals. The results revealed no significant relationship between Schoenfeld residuals for NS and follow-up time $(\mathrm{p}=0.29)$ in the model evaluating PDsPS risk.

The incidence rates of PDsPS were 0.75/0.56 per 1000 person-years without comorbidities, 2.03/1.57 per 1000 person-years with one comorbidity and 5.23/4.60 per 1000 person-years with more than two comorbidities in the combined NS and non-NS cohorts. The multivariable Cox model estimated the aHR of PDsPS to be 1.66 (95\% CI 1.43 to 1.94 ) without comorbidities, 1.63 (95\% CI 1.28 to 2.08) with one comorbidity and 1.35 (95\% CI 1.12 to $1.63)$ with more than two comorbidities in the NS cohort compared with the non-NS cohort (table 3 ).

\section{DISCUSSION}

This is the first nationwide and population-based cohort study to investigate the association between NS and PDsPS. After adjustment for sex, age and comorbidities, patients with NS were 1.51 times more likely to be diagnosed with PDsPS than were those without NS. Cases of a PDsPS diagnosis with immunosuppressant therapy or rituximab and prednisolone biological therapy were rare in our study, and we considered only the HR in patients who did not undergo these treatments to prevent the confounding effects of immunotherapy on PDsPS risk. ${ }^{28}$ Although cases in which the onset of PDsPS occurred at an age younger than 40 were less than 5\% and PDsPS in such patients might be associated with genetic defects, ${ }^{29}$ the mean age

Table 3 Nephrotic syndrome accompanied with different amounts of comorbidities all increased the incidence and HR of Parkinson's disease and secondary parkinsonism

\begin{tabular}{|c|c|c|c|c|c|c|c|c|}
\hline \multirow[b]{3}{*}{ Variable } & \multicolumn{6}{|c|}{ Nephrotic syndrome } & \multirow{3}{*}{$\begin{array}{l}\text { Crude HR } \\
(95 \% \mathrm{Cl})\end{array}$} & \multirow{3}{*}{$\begin{array}{l}\text { Adjusted HR } \\
(95 \% \mathrm{Cl})\end{array}$} \\
\hline & \multicolumn{3}{|l|}{ Yes } & \multicolumn{3}{|l|}{ No } & & \\
\hline & Event & PY & Rate & Event & PY & Rate & & \\
\hline 0 & 9 & 11967 & 0.75 & 35 & 62251 & 0.56 & $1.34(1.13 \text { to } 1.58)^{\star \star \star}$ & $1.66(1.43 \text { to } 1.94)^{\star \star \star}$ \\
\hline 1 & 10 & 4917 & 2.03 & 26 & 16535 & 1.57 & $1.29(1.01 \text { to } 1.66)^{*}$ & $1.63(1.28 \text { to } 2.08)^{\star \star \star}$ \\
\hline
\end{tabular}

†Patients with any one of the comorbidities (diabetes, hypertension, hyperlipidaemia, coronary artery disease, head injury, COPD, depression, stroke, dementia and epilepsy).

Adjusted HR, HR adjusted for age and immunosuppressant therapy and the biological therapy rituximab with prednisolone; crude HR, relative HR; PY, person-years; rate, incidence rate per 1000 person-years.

${ }^{\star} P<.05,{ }^{* \star} P<.01,{ }^{* \star *} P<.001$ 
in this study was only 50.2 years. One possible explanation for this was that the current study was designed to target NS patients without CKD, and these patients were seldom the elderly because the prevalence of CKD increases with age. Stroke and dementia can cause sPS; however, when we excluded patients with these conditions, significant correlations between NS and PDsPS remained. In addition, although uncommon, epilepsy in comorbidities with PD might influence the progression of PD. ${ }^{30}$ We found that patients with a poor control of epilepsy had a higher risk of $\mathrm{PD}$, and therefore listed epilepsy as a potential comorbidity. We observed that patients with NS exhibited a significantly higher risk of PDsPS incidence than did those without NS, irrespective of sex, age ( $<65$ years) and comorbidities. These results support our observation that NS independently influences PDsPS.

$\mathrm{PD}$ is a progressive neurodegenerative disorder characterised by both motor and non-motor manifestations such as bradykinesia, resting tremor, rigidity, neuropsychiatric symptoms, postural instability, autonomic dysfunction and sleep difficulties. ${ }^{31}$ Pathogenesis of PD is associated with a loss of dopaminergic neurons in the substantia nigra pars compacta, which can be affected by intracranial inflammation. ${ }^{18}$ It has been hypothesised that patients with NS have an increased risk of PDsPS. Furthermore, the aetiology of PDsPS may be associated with a blood-brain barrier impairment-related increase in neuroinflammation in the brain. Viruses can invade the impaired blood-brain barrier to reach the substantia nigra, which can directly cause cell death and possibly induce a cytokine imbalance in the brain resulting in microglia activation and neuron apoptosis. ${ }^{32}$ A study has indicated that neuroinflammation can be also caused by non-CNS infections. ${ }^{33}$ Tuberculosis, a common chronic infection of the lung that induces proinflammatory responses, also increases the risk of PD. It is possible that tuberculosis increases sPS risk, as similar diagnostic criteria were used (ICD-9 code 332) ${ }^{34}$ Chronic inflammation of the brain could elevate the risk of PDsPS, and blood-brain barrier impairment might allow more inflammatory cytokines, proinflammatory factors or viruses to enter the brain and cause inflammation.

In a previous study, the Alzheimer's disease risk factor CD2-associated protein (CD2AP) was shown to play a role in maintaining glomerular integrity in the kidney. ${ }^{35}$ Immune-histochemical studies have revealed that CD2AP was localised primarily in cerebrovascular endothelial cells in the brain. ${ }^{36}{ }^{37}$ We determined the association among the CD2AP, blood-brain barrier integrity, NS and Alzheimer's disease risk. Previous studies have proposed that inflammation plays a role in the pathogenesis of PD, and we posit that this is also likely to be the case in sPS. ${ }^{38} 39$ In previous studies, risk factors for late-onset Alzheimer's disease have been implicated in blood-brain barrier integrity or pathways, including APOE, PICALM, INPP5D and $C L U{ }^{40}$ The relationship between PDsPS and NS must be clarified in the future.

In another study of 186 patients, impaired renal function and proteinuria, which are major characteristics of NS, were associated with cognitive impairment and dementia. The urinary protein:creatinine ratio was significantly higher in patients with PD dementia and dementia with Lewy bodies than in healthy controls or in those with PD and mild impairment or normal cognition. The urinary protein:creatinine ratio was also correlated with white matter hyperintensities on MRI scans and abnormal neuropsychological test results. ${ }^{19}$ The Lewy bodies dementia could be characterised by PS. ${ }^{41}$ Therefore, the higher urinary protein:creatinine ratio might elevate the risk of PD and PS. Proteinuria can cause hypoproteinemia, reduce the cytokine level or impair related pathways leading to the degeneration of dopaminergic neurons. In previous studies, proteinuria was associated with cognitive impairment and an increased volume of white matter hyperintensities, which are both associated with vascular brain injury. ${ }^{21}$ Both neuron degeneration and vascular damage in the brain have been associated with several neurodegenerative diseases, such as PD or PS. ${ }^{242}$ Our results reveal that NS not only causes proteinuria to increase the risk of dementia but also elevates the risk of PDsPS.

This study has several potential limitations. First, the National Health Insurance Research Database (NHIRD) lacks information on patient behaviours and other risk factors, such as smoking, obesity, alcoholism, family history, exercise, occupation and lifestyle. Therefore, we could not ensure the possible pathogenesis of NS in patients with PDsPS. In addition, medications being used by patients might also contribute to parkinsonism ${ }^{43}$; however, details regarding prescribed medications were not included in the NHIRD. Second, because several clinical variables, such as laboratory data, imaging results, urine protein and creatinine levels, and protein loss reports were unavailable in our study, we could not assess the severity of NS in the study patients. Third, the diagnosis of PDsPS was based on clinical presentation, because neuroimaging findings are not available in the NHIRD. Fourth, the patients of PDsPS were primarily diagnosed by neurologists in Taiwan, but we cannot confirm how many patients were diagnosed by general physicians. Fifth, because our study included predominantly Asian patients, the results may not be generalisable to other ethnic populations. Sixth, PD and sPS diagnoses sometimes overlap due to possible diagnostic bias towards PD in certain clinics and the inability to confirm the underlying cause of the disease. Finally, as is common with a retrospective cohort study, compared with a randomised trial, there is an increased risk of potential bias after adjustment for confounding variables.

\section{CONCLUSIONS}

This study demonstrated that patients with NS exhibited a 1.51-fold increase in the risk of PDsPS compared with those without NS in a nationwide, population-based database. Although evidence of the association between 
NS and PDsPS is limited, our results demonstrated that the patients with NS exhibited an increased risk of PDsPS. NS appeared to coexist with CKD in some patients, but this occurred more often in the patients without kidney disease or with early-stage renal disease and normal creatinine levels. CKD elevates the risk of $\mathrm{PD}$ in the presence of uraemia, similar to the effects on PDsPS exerted by NS in our study. Based on these findings, physicians should begin the management of renal injury early, and not delay medical care until chronic renal failure status, due to the possibility that NS might cause brain damage. The risk of PDsPS in patients with NS must be considered and adequate treatment must be prescribed to prevent PDsPS development.

\section{Author affiliations}

${ }^{1}$ Division of Rheumatology, Immunology and Allergy, Department of Medicine, TriService General Hospital, National Defense Medical Center, Taipei, Taiwan ${ }^{2}$ Division of Rheumatology, Immunology and Allergy, Department of Medicine, Kaohsiung Armed Forces General Hospital, Kaohsiung, Taiwan

${ }^{3}$ Department of Health Promotion and Health Education, National Defense Medical Center, Taipei, Taiwan

${ }^{4}$ School of Medicine, China Medical University, Taichung, Taiwan

${ }^{5}$ Management Office for Health Data, China Medical University Hospital, Taichung, Taiwan

${ }^{6}$ Department of Nuclear Medicine and PET Center, China Medical University Hospital, Taichung, Taiwan

${ }^{7}$ Graduate Institute of Clinical Medicine Science and School of Medicine, College of Medicine, China Medical University, Taichung, Taiwan

${ }^{8}$ Graduate Institute of Chinese Medicine, China Medical University, Taichung, Taiwan ${ }^{9}$ Molecular and Genomic Epidemiology Center, China Medical University Hospital, Taichung, Taiwan

Contributors T-YY and F-CL contributed equally. Conceived and designed the experiments: Z-HH, F-CL, T-YY. Contributed reagents, materials and analytical tools: C-HK, T-YY. Conducted experiments, analysed the data: C-LL, C-HK, T-YY. Drafted the manuscript and approved the manuscript: Z-HH, Y-CC, H-CC, C-LL, C-HK, H-YL, T-YY and F-CL.

Funding This study is supported in part by Taiwan Ministry of Health and Welfare Clinical Trial and Research Center of Excellence (MOHW104-TDU-B-212-113002); China Medical University Hospital, Academia Sinica Taiwan Biobank, Stroke Biosignature Project (BM104010092); NRPB Stroke Clinical Trial Consortium (MOST 103-2325-B-039-006); Ministry of Science and Technology (MOST 105-2314-B-016-052-MY2); Tseng-Lien Lin Foundation, Taichung, Taiwan; Taiwan Brain Disease Foundation, Taipei, Taiwan; Katsuzo and Kiyo Aoshima Memorial Funds, Japan; and CMU under the Aim for Top University Plan of the Ministry of Education, Taiwan. No additional external funding was received for this study.

Competing interests None declared.

Patient consent Not required.

Ethics approval This study was approved by the Ethics Review Board of China Medical University and Hospital in Taiwan (CMUH-104-REC2-115).

Provenance and peer review Not commissioned; externally peer reviewed.

Data sharing statement Data are available. Please contact corresponding author.

Open access This is an open access article distributed in accordance with the Creative Commons Attribution Non Commercial (CC BY-NC 4.0) license, which permits others to distribute, remix, adapt, build upon this work non-commercially, and license their derivative works on different terms, provided the original work is properly cited, appropriate credit is given, any changes made indicated, and the use is non-commercial. See:Chttp://creativecommons.org/licenses/by-nc/4.0/.

\section{REFERENCES}

1. Dickson DW. Parkinson's disease and parkinsonism: neuropathology. Cold Spring Harb Perspect Med 2012;2:a009258.
2. Liang CY, Yeh YC, Lee CJ, et al. Flunarizine and the risk of parkinsonism in a newly diagnosed type 2 diabetic population in Taiwan: A nested case-control study. J Clin Neurosci 2018;50:281-6.

3. Rektor I, Bohnen NI, Korczyn AD, et al. An updated diagnostic approach to subtype definition of vascular parkinsonism Recommendations from an expert working group. Parkinsonism Relat Disord 2018;49:9-16.

4. Schneider SA, Alcalay RN. Neuropathology of genetic synucleinopathies with parkinsonism: Review of the literature. Mov Disord 2017;32:1504-23.

5. Chen L, Mo M, Li G, et al. The biomarkers of immune dysregulation and inflammation response in Parkinson disease. Trans Neurodegener 2016;5:eCollection:16.

6. de Lau LM, Breteler MM. Epidemiology of Parkinson's disease. Lancet Neurol 2006;5:525-35.

7. Liu WM, Wu RM, Lin JW, et al. Time trends in the prevalence and incidence of Parkinson's disease in Taiwan: A nationwide, population-based study. J Formos Med Assoc 2016;115:531-8.

8. Wang IK, Lin CL, Wu YY, et al. Increased risk of Parkinson's disease in patients with end-stage renal disease: a retrospective cohort study. Neuroepidemiology 2014;42:204-10.

9. Gardner RC, Burke JF, Nettiksimmons J, et al. Traumatic brain injury in later life increases risk for Parkinson disease. Ann Neurol 2015;77:987-95.

10. Gustafsson H, Nordström A, Nordström P. Depression and subsequent risk of Parkinson disease: A nationwide cohort study. Neurology 2015;84:2422-9.

11. Park HJ, Lee KW, Park ES, et al. Dysregulation of protein phosphatase $2 \mathrm{~A}$ in parkinson disease and dementia with lewy bodies. Ann Clin Transl Neurol 2016;3:769-80.

12. Jellinger KA. Prevalence of cerebrovascular lesions in Parkinson's disease. A postmortem study. Acta Neuropathol 2003;105:415-9.

13. Li CH, Chen WC, Liao WC, et al. The association between chronic obstructive pulmonary disease and Parkinson's disease: a nationwide population-based retrospective cohort study. QJM 2015;108:39-45.

14. Bohnen NI, Kotagal V, Müller MLTM, et al. Diabetes mellitus is independently associated with more severe cognitive impairment in Parkinson disease. Parkinsonism Relat Disord 2014;20:1394-8.

15. Qiu C, Hu G, Kivipelto M, et al. Association of blood pressure and hypertension with the risk of Parkinson disease: the National FINRISK Study. Hypertension 2011;57:1094-100.

16. Tufekci KU, Meuwissen R, Genc S, et al. Inflammation in Parkinson's disease. Adv Protein Chem Struct Biol 2012;88:69-132.

17. Nishi S, Ubara $Y$, Utsunomiya $Y$, et al. Evidence-based clinical practice guidelines for nephrotic syndrome 2014. Clin Exp Nephrol 2016;20:342-70.

18. Elias MF, Dore GA, Davey A. Kidney disease and cognitive function. Contrib Nephrol 2013;179:42-57.

19. Sasaki $Y$, Marioni R, Kasai M, et al. Chronic kidney disease: a risk factor for dementia onset: a population-based study. The Osaki-Tajiri Project. J Am Geriatr Soc 2011;59:1175-81.

20. Gorriz JL, Martinez-Castelao A. Proteinuria: detection and role in native renal disease progression. Transplant Rev 2012;26:3-13.

21. Barzilay JI, Fitzpatrick AL, Luchsinger J, et al. Albuminuria and dementia in the elderly: a community study. Am J Kidney Dis 2008:52:216-26.

22. Jassal SK, Kritz-Silverstein D, Barrett-Connor E. A prospective study of albuminuria and cognitive function in older adults: the Rancho Bernardo study. Am J Epidemiol 2010;171:277-86.

23. Ys O, Kim JS, Park HE, et al. Association between urine protein/ creatinine ratio and cognitive dysfunction in Lewy body disorders. $J$ Neurol Sci 2016;362:258-62.

24. Weiner DE, Bartolomei K, Scott T, et al. Albuminuria, cognitive functioning, and white matter hyperintensities in homebound elders. Am J Kidney Dis 2009;53:438-47.

25. Database NHIR. Taiwan. http://nhird.nhri.org.tw/en/index.html (cited in 2015).

26. Sh W, Chuang E, Chuang TY, et al. A Nationwide PopulationBased Cohort Study of Migraine and Organic-Psychogenic Erectile Dysfunction. Medicine 2016;95:e3065.

27. Lin JC, Lin CS, Hsu CW, et al. Association Between Parkinson's Disease and Inflammatory Bowel Disease: a Nationwide Taiwanese Retrospective Cohort Study. Inflamm Bowel Dis 2016;22:1049-55.

28. Liu FC, Huang WY, Lin TY, et al. Inverse Association of Parkinson Disease With Systemic Lupus Erythematosus: A Nationwide Population-based Study. Medicine (Baltimore). 2015;94:e 2097.

29. Tysnes O-B, Storstein A. Epidemiology of Parkinson's disease. J Neural Transm 2017;124:901-5.

30. Son AY, Biagioni MC, Kaminski D, et al. Parkinson's Disease and Cryptogenic Epilepsy. Case Rep Neurol Med 2016;2016:3745631. 
31. Cusso ME, Donald KJ, Khoo TK. The Impact of Physical Activity on Non-Motor Symptoms in Parkinson's Disease: A Systematic Review. Front Med 2016;3:35.

32. Wy W, Kang KH, Chen SL, et al. Hepatitis $\mathrm{C}$ virus infection: a risk factor for Parkinson's disease. J Viral Hepat 2015;22:784-91.

33. Collins LM, Toulouse A, Connor TJ, et al. Contributions of central and systemic inflammation to the pathophysiology of Parkinson's disease. Neuropharmacology 2012;62:2154-68.

34. Shen $\mathrm{CH}$, Chou CH, Liu FC, et al. Association Between Tuberculosis and Parkinson Disease: A Nationwide, Population-Based Cohort Study. Medicine 2016;95:e2883.

35. Shih NY, Li J, Karpitskii V, et al. Congenital nephrotic syndrome in mice lacking CD2-associated protein. Science 1999;286:312-5.

36. Li C, Ruotsalainen V, Tryggvason K, et al. CD2AP is expressed with nephrin in developing podocytes and is found widely in mature kidney and elsewhere. Am J Physiol Renal Physiol 2000;279:F78 5-F792.
37. Lehtonen S, Tienari J, Londesborough A, et al. CD2-associated protein is widely expressed and differentially regulated during embryonic development. Differentiation 2008;76:506-17.

38. Bohara M, Kambe Y, Nagayama T, et al. C-type natriuretic peptide modulates permeability of the blood-brain barrier. J Cereb Blood Flow Metab 2014;34:589-96.

39. Obermeier B, Daneman R, Ransohoff RM. Development, maintenance and disruption of the blood-brain barrier. Nat Med 2013;19:1584-96.

40. Rosenthal SL, Kamboh MI. Late-Onset Alzheimer's Disease Genes and the Potentially Implicated Pathways. Curr Genet Med Rep 2014;2:85-101.

41. Wakabayashi K, Tanji K, Odagiri S, et al. The Lewy body in Parkinson's disease and related neurodegenerative disorders. Mol Neurobiol 2013;47:495-508

42. Guan J, Pavlovic D, Dalkie N, et al. Vascular degeneration in Parkinson's disease. Brain Pathol 2013;23:154-64.

43. Rochon PA, Stukel TA, Sykora K, et al. Atypical Antipsychotics and Parkinsonism. Arch Intern Med 2005;165:1882-8. 